\title{
Analysis of the risk factors of acute kidney injury after total hip or knee replacement surgery
}

\author{
Yoo Jin Lee, Bong Soo Park, Sihyung Park, Jin Han Park, Il Hwan Kim, Junghae Ko, Yang Wook Kim
}

Department of Internal Medicine, Inje University Haeundae Paik Hospital, Inje University College of Medicine, Busan, Korea

Received: July 1, 2020

Revised: September 15, 2020

Accepted: September 16, 2020

Corresponding author:

Yang Wook Kim, MD

Department of Internal Medicine,

Inje University Haeundae Paik

Hospital, Inje University College of

Medicine, 875 Haeun-daero,

Haeundae-gu, Busan 48108, Korea

Tel: +82-51-797-3324

Fax: +82-51-797-3282

E-mail:kyw8625@chol.com
Background: Postoperative acute kidney injury (AKI), which increases the risk of postoperative morbidity and mortality, poses a major concern to surgeons. We conducted this study to analyze the risk factors associated with the occurrence of AKI after orthopedic surgery.

Methods: This was a retrospective study that included 351 patients who underwent total hip or knee replacement surgery at Inje University Haeundae Paik Hospital between January 2012 and December 2016.

Results: AKI occurred in $13(3.7 \%)$ of the 351 patients. The patients' preoperative estimated glomerular filtration rate (eGFR) was $66.66 \pm 34.02 \mathrm{~mL} / \mathrm{min} / 1.73 \mathrm{~m}^{2}$ in the AKI group and $78.07 \pm 21.23 \mathrm{~mL} / \mathrm{min} / 1.73 \mathrm{~m}^{2}$ in the non-AKI group. The hemoglobin levels were $11.21 \pm 1.65 \mathrm{~g} /$ $\mathrm{dL}$ in the AKI group and $12.39 \pm 1.52 \mathrm{~g} / \mathrm{dL}$ in the non-AKI group. Hemoglobin level was related to increased risk of AKI (odds ratio [OR], $0.13 ; 95 \%$ confidence interval [Cl], $0.02-0.68 ; p=0.016$ ). Administration of crystalloid or colloid fluid alone and the perioperative amount of fluid did not show any significant relationship with AKI. Further analysis of the changes in eGFR was performed using a cutoff value of 7.54. The changes in eGFR were significantly related to decreased risk of AKI (OR, $0.74 ; 95 \% \mathrm{Cl}, 0.61-0.89 ; p=0.002)$.

Conclusion: Renal function should be monitored closely after orthopedic surgery if patients have chronic kidney disease and low hemoglobin level. Predicting the likelihood of AKI occurrence, early treatment of high-risk patients, and monitoring perioperative laboratory test results, including eGFR, will help improve patient prognosis.

Keywords: Acute kidney injury; Orthopedic procedures; Postoperative complications; Risk factors

\section{Introduction}

Postoperative acute kidney injury (AKI), which increases the risk of postoperative morbidity and mortality, poses a major concern to surgeons $[1,2]$. Previous studies reported a $9.1 \%$ risk of AKI after elective or emergency orthopedic surgical procedures $[3,4]$. According to a study conducted in patients who underwent any type of inpatient operative procedure in the United States, hospital mortality showed a high correlation with postoperative AKI. In patients with AKI, the lengths of stays in the intensive care unit ( 6 days vs. 2 days) and hospital (12 days vs. 5 days) were longer and the risk-adjusted average cost of care was higher than those in patients without AKI (US dollar [USD] 42,600 vs. USD 26,700) [5].

Several perioperative risk factors are associated with the development of AKI. The major perioperative risk factors include underlying chronic kidney disease (CKD), diabetes, and congestive heart failure during cardiac surgery [6]. However, little is known about the occurrence of postoperative AKI after orthopedic surgery. Patients undergoing orthopedic surgery are expected to have

Copyright (C) 2021 Yeungnam University College of Medicine

This is an Open Access article distributed under the terms of the Creative Commons Attribution Non-Commercial License (http://creativecommons.org/licenses/by-nc/4.0/) which permits unrestricted non-commercial use, distribution, and reproduction in any medium, provided the original work is properly cited. 
a high probability of renal dysfunction because of comorbidities, potential high volume of blood loss, and development of perioperative infection. Recently, the number of orthopedic surgeries being performed has been increasing [7] along with the aging of the Korean population [8], which leads to a high number of high-risk Korean patients. Thus, we conducted this study to analyze the risk factors associated with the occurrence of AKI after orthopedic surgery to provide ample information that will aid the effective management of patients, such as elderly patients who have undergone orthopedic surgery.

\section{Materials and methods}

\section{Patients}

This study was approved by the Institutional Review Board (IRB) of the Inje University Haeundae Paik Hospital (IRB No: 2020-08018). Written informed consent was obtained from the study subjects. This retrospective study included 351 patients who underwent total hip or knee replacement surgery in Inje University Haeundae Paik Hospital between January 2012 and December 2016. Age, sex, preoperative glomerular filtration rate (GFR), postoperative GFR, drugs used (nonsteroidal anti-inflammatory drugs, angiotensin-converting-enzyme inhibitors, and statins), number of prescribed drugs (total number of drugs prescribed and taken at the hospital before surgery), albumin level ( $\mathrm{g} / \mathrm{dL}$ ), hemoglobin level $(\mathrm{g} / \mathrm{dL})$, pro-B-type natriuretic peptide level $(\mathrm{pg} / \mathrm{mL})$, ejection fraction, and perioperative amount of fluid (the total amount of fluid administered in 3 days, including the day before surgery), types of fluid administered during surgery, presence of diabetes and hypertension, and presence of CKD were assessed.

\section{Definition}

AKI was defined as a postoperative creatinine level of $\geq 0.3 \mathrm{mg} / \mathrm{dL}$ [9], and CKD was defined as a GFR of $<60 \mathrm{~mL} / \mathrm{min} / 1.73 \mathrm{~m}^{2}$, calculated using the Chronic Kidney Disease Epidemiology Collaboration equation [10]. The postoperative estimated GFR (eGFR) was based on value obtained on the third day after surgery. The change in eGFR from baseline was indicated as $\triangle \mathrm{eGFR}$.

\section{Statistical analyses}

The study data are presented as frequencies with percentages for categorical variables and means \pm standard deviations for continuous variables. The effect of the independent variables on the response variables was analyzed using multivariate logistic regression, and the statistically significant variables were selected using a backward elimination method with a 0.05 alpha level. Differences in the characteristics of the study participants were compared across subgroups by using the chi-square or Fisher exact test for categorical variables and the independent $t$-test or Mann-Whitney $U$-test for continuous variables. The Shapiro-Wilk test was used to check if the data distribution was normal. Univariate and multivariate analyses were performed using logistic regression to identify prognostic factors that are independently related to AKI. A receiver-operating characteristic curve was used to assess the sensitivity and specificity of the change in eGFR from baseline. Positive and negative predictive values were also calculated. All statistical analyses were performed using the IBM SPSS version 24.0 (IBM Corp., Armonk, NY, USA) and MedCalc version 11.6.0 software (MedCalc Software Ltd., Ostend, Belgium). A $p$-value of $<0.05$ was considered statistically significant.

\section{Results}

The baseline characteristics of the study patients are shown in Table 1. Overall, AKI occurred in 13 (3.7\%) of the 351 patients. The mean ages of the study participants in the AKI and non-AKI groups were $67.92 \pm 13.49$ years and $66.34 \pm 11.03$ years, respectively. Of the study population, 269 patients (76.6\%) were women, and 66 (18.8\%) had CKD. The patients' preoperative eGFR was $66.66 \pm 34.02 \mathrm{~mL} / \mathrm{min} / 1.73 \mathrm{~m}^{2}$ in the AKI group and $78.07 \pm 21.23 \mathrm{~mL} / \mathrm{min} / 1.73 \mathrm{~m}^{2}$ in the non-AKI group. The albumin level was $3.31 \pm 0.32 \mathrm{~g} / \mathrm{dL}$ in the AKI group and $3.67 \pm 0.42$ $\mathrm{g} / \mathrm{dL}$ in the non-AKI group. The hemoglobin level was $11.21 \pm 1.65$ $\mathrm{g} / \mathrm{dL}$ in the AKI group and $12.39 \pm 1.52 \mathrm{~g} / \mathrm{dL}$ in the non-AKI group. The $\triangle \mathrm{eGFR}$ was significantly related to decreased risk of AKI (odds ratio [OR], 0.74; 95\% confidence interval [CI], 0.61$0.89 ; p=0.002)$; Hemoglobin level showed statistically significant results with acute renal injury (OR, 0.13 ; 95\% CI, 0.02-0.68; $p=0.016)$. Lower hemoglobin level was associated with more acute renal injury. Regarding the administration of perioperative fluid therapy, eight (3.7\%) of the 219 patients in the crystalloid group and five (3.8\%) of the 132 patients in the colloid group developed AKI. Administration of crystalloid or colloid fluid alone did not show any significant association with the development of AKI. The perioperative amount of fluid was 1,330.77 \pm 606.06 $\mathrm{mL}$ in the AKI group and $1,539.85 \pm 942.03 \mathrm{~mL}$ in the non-AKI group, showing no statistically significant difference (Table 1 ).

The multivariate analysis revealed that the other variables were not related to AKI, although some variables showed significance after the univariate analysis. The $\triangle \mathrm{eGFR}$ was further analyzed using a cutoff value of 7.54. The sensitivity, specificity, false-negative rate, false-positive rate, positive predicted value, and negative predicted value of $\triangle \mathrm{eGFR}$ for predicting AKI were $100 \%, 89.64 \%$, $0.00 \%, 10.36 \%, 27.08 \%$, and $100 \%$, respectively. The concordance 
Table 1. Patients' demographic and clinical variables across groups

\begin{tabular}{|c|c|c|c|c|c|c|c|}
\hline \multirow{2}{*}{ Variable } & \multicolumn{3}{|c|}{ Acute kidney injury } & \multicolumn{2}{|c|}{ Univariate logistic regression } & \multicolumn{2}{|c|}{ Multivariate logistic regression } \\
\hline & Yes $(n=13)$ & No $(n=338)$ & $p$-value & OR $(95 \% \mathrm{Cl})$ & $p$-value & OR $(95 \% \mathrm{Cl})$ & $p$-value \\
\hline Age $(y r)$ & $67.92 \pm 13.49$ & $66.34 \pm 11.03$ & $0.280^{b)}$ & $1.01(0.96-1.07)$ & 0.614 & - & - \\
\hline \multicolumn{8}{|l|}{ Sex } \\
\hline Male & $3(23.1)$ & $79(23.4)$ & $>0.999^{c)}$ & $0.98(0.26-3.66)$ & 0.980 & - & - \\
\hline Female & $10(76.9)$ & $259(76.6)$ & & & & - & - \\
\hline \multicolumn{8}{|l|}{ Diabetes mellitus } \\
\hline Yes & $2(15.4)$ & 66 (19.5) & $>0.999^{c)}$ & $0.75(0.16-3.46)$ & 0.712 & - & - \\
\hline No & $11(84.6)$ & $272(80.5)$ & & 1.00 & & - & - \\
\hline \multicolumn{8}{|l|}{ Hypertension } \\
\hline Yes & $11(84.6)$ & $182(53.8)$ & $0.029^{d)}$ & $4.71(1.03-21.59)$ & 0.046 & - & - \\
\hline No & $2(15.4)$ & $156(46.2)$ & & 1.00 & & - & - \\
\hline \multicolumn{8}{|l|}{ CKD } \\
\hline Yes & $9(69.2)$ & 57 (16.9) & $<0.001^{\mathrm{c}}$ & 11.09 (3.30-37.26) & $<0.001$ & - & - \\
\hline No & $4(30.8)$ & $281(83.1)$ & & 1.00 & & - & - \\
\hline \multicolumn{8}{|l|}{ ACEi } \\
\hline Yes & $4(30.8)$ & $110(32.5)$ & $>0.999^{c)}$ & $0.88(0.27-2.94)$ & 0.842 & - & - \\
\hline No & $9(69.2)$ & $219(64.8)$ & & 1.00 & & - & - \\
\hline \multicolumn{8}{|l|}{ NSAIDs } \\
\hline Yes & $2(15.4)$ & $50(14.8)$ & $>0.999^{c)}$ & $1.01(0.22-4.72)$ & 0.985 & - & - \\
\hline No & $11(84.6)$ & $279(82.5)$ & & 1.00 & & - & - \\
\hline \multicolumn{8}{|l|}{ Anesthesia } \\
\hline Spinal & $11(84.6)$ & $267(79.0)$ & $>0.999^{c)}$ & $1.46(0.32-6.75)$ & 0.626 & - & - \\
\hline General & $2(15.4)$ & $71(21.0)$ & & 1.00 & & - & - \\
\hline During operative bleeing $(\mathrm{mL})$ & $155.77 \pm 149.41$ & $227.57 \pm 260.51$ & $0.558^{b)}$ & $1.00(1.00-1.00)$ & 0.325 & - & - \\
\hline Operation time (min) & $173.08 \pm 25.78$ & $186.88 \pm 41.10$ & $0.267^{b)}$ & $0.99(0.97-1.01)$ & 0.231 & - & - \\
\hline \multicolumn{8}{|l|}{ Type of surgery } \\
\hline Total knee replacement & $9(69.2)$ & $229(67.8)$ & $1.000^{\mathrm{c})}$ & $1.07(0.32-3.55)$ & 0.911 & - & - \\
\hline Total hip replacement & $4(30.8)$ & $109(32.2)$ & & 1.00 & & - & - \\
\hline Preoperative GFR (mL/min/1.73 $\left.\mathrm{m}^{2}\right)$ & $66.66 \pm 34.02$ & $78.07 \pm 21.23$ & $0.043^{b)}$ & $0.97(0.95-1.00)$ & 0.058 & - & - \\
\hline$\triangle \mathrm{eGFR}$ & $-29.47 \pm 18.10$ & $10.89 \pm 14.93$ & $<0.001^{\mathrm{e})}$ & $0.82(0.75-0.90)$ & $<0.001$ & $0.74(0.61-0.89)$ & 0.002 \\
\hline Albumin (g/dL) & $3.31 \pm 0.32$ & $3.67 \pm 0.42$ & $0.001^{\mathrm{b})}$ & $0.13(0.03-0.49)$ & 0.003 & - & - \\
\hline Hemoglobin $(\mathrm{g} / \mathrm{dL})$ & $11.21 \pm 1.65$ & $12.39 \pm 1.52$ & $0.015^{b)}$ & $0.61(0.43-0.88)$ & 0.008 & $0.13(0.02-0.68)$ & 0.016 \\
\hline Postopeative CRP (mg/L) & $13.73 \pm 8.09$ & $13.39 \pm 7.18$ & $0.786^{b)}$ & $1.01(0.93-1.09)$ & 0.870 & - & - \\
\hline Total $\mathrm{CO}_{2}(\mathrm{mmol} / \mathrm{L})$ & $24.28 \pm 2.82$ & $26.53 \pm 3.22$ & $0.019^{b)}$ & $0.78(0.64-0.95)$ & 0.013 & - & - \\
\hline Ejection fraction (\%) & $65.04 \pm 6.99$ & $64.66 \pm 4.09$ & $0.354^{b)}$ & $1.02(0.87-1.19)$ & 0.788 & - & - \\
\hline Hospital stay (day) & $24.82 \pm 6.59$ & $23.55 \pm 12.14$ & $0.104^{b)}$ & $1.01(0.96-1.06)$ & 0.731 & - & - \\
\hline No. of prescribed drug & $7.36 \pm 3.93$ & $4.11 \pm 3.47$ & $0.006^{b)}$ & $1.23(1.07-1.43)$ & 0.005 & - & - \\
\hline $\operatorname{proBNP}(\mathrm{ng} / \mathrm{L})$ & $248.57 \pm 274.08$ & $235.01 \pm 409.40$ & $0.511^{b)}$ & $1.00(1.00-1.00)$ & 0.954 & - & - \\
\hline \multicolumn{8}{|l|}{ Fluid therapy } \\
\hline Crystalloid & $8(61.5)$ & $211(62.4)$ & $1.000^{\mathrm{c})}$ & 1.00 & & - & - \\
\hline Colloid & $5(38.5)$ & $127(37.6)$ & & $1.04(0.33-3.24)$ & 0.948 & - & - \\
\hline Perioperative amount of fluid (mL) & $1,330.77 \pm 606.06$ & $1,539.85 \pm 942.03$ & $0.479^{b)}$ & $1.00(1.00-1.00)$ & 0.425 & - & - \\
\hline
\end{tabular}

Values are presented as mean \pm standard deviation or number (\%).

CKD, chronic kidney disease; ACEi, angiotensin-converting enzyme inhibitor; NSAIDs, nonsteroidal anti-inflammatory drugs; eGFR, estimated glomerular filtration rate; $\triangle \mathrm{eGFR}$, change in eGFR at postoperative from baseline; CRP, C-reactive protein; proBPN, pro-B-type natriuretic peptide.

${ }^{a}$ In a multivariate logistic regression, the statistically significant variables in the univariate analyses were included. Then, categorical variables that have less frequency in a certain subgroup such as hypertension and CKD were excluded since the estimated odds ratio goes infinity and is not estimable. Finally, the statistically significant variables were selected in a backward elimination method with 0.05 alpha level.

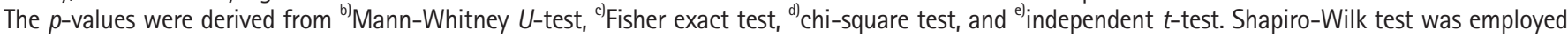
for test of normality assumption. 
between a $\triangle \mathrm{eGFR}$ of -7.54 and the occurrence of AKI was good $(316 / 351,90.03 \%)$ (Table 2, Fig. 1).

\section{Discussion}

This study involved the analysis of the risk factors for AKI in pa-

Table 2. Comparison of efficacies of $\triangle \mathrm{eGFR}$ for detecting the acute kidney injury

\begin{tabular}{|c|c|c|}
\hline \multirow{2}{*}{ Variable } & \multicolumn{2}{|c|}{ Cut-point value of $\triangle \mathrm{eGFR}$} \\
\hline & $\leq-7.54$ & $>-7.54$ \\
\hline \multicolumn{3}{|l|}{ Acute kidney injury } \\
\hline Yes & 13 & 0 \\
\hline No & 35 & 303 \\
\hline AUC ( $p$-value) & \multicolumn{2}{|c|}{$0.98(<0.001)$} \\
\hline Sensitivity (\%) & \multicolumn{2}{|c|}{$13 / 13(100)$} \\
\hline Specificity (\%) & \multicolumn{2}{|c|}{ 303/338 (89.64) } \\
\hline FNR, 100\%-sensitivity & \multicolumn{2}{|c|}{ 0/13 (0) } \\
\hline FPR, 100\%-specificity & \multicolumn{2}{|c|}{ 35/338 (10.36) } \\
\hline PPV (\%) & \multicolumn{2}{|c|}{ 13/48 (27.08) } \\
\hline NPV (\%) & \multicolumn{2}{|c|}{ 303/303 (100) } \\
\hline
\end{tabular}

$\triangle \mathrm{eGFR}$, change in estimated glomerular filtration rate at postoperative from baseline; AUC, area under the curve; sensitivity, proportion of patients with acute kidney injury who had a lower than -7.54 for $\triangle \mathrm{eGFR}$; specificity, proportion of patients without acute kidney injury who had a higher than -7.54 for $\triangle \mathrm{eGFR}$; FNR, false-negative rate; FPR, false-positive rate; PPV, positive predicted value; NPV, negative predicted value. Concordance between $\triangle \mathrm{eGFR}$ with -7.54 and acute kidney injury was $\operatorname{good}(316 / 351,90.03 \%)$.

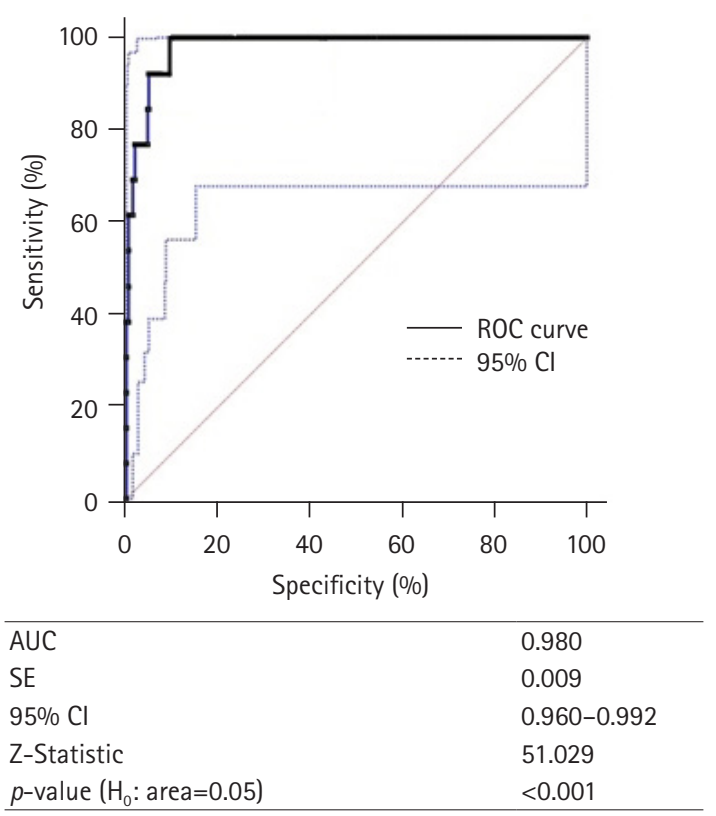

Fig. 1. Efficacy rates of changes in estimated glomerular filtration rate ( $\triangle \mathrm{eGFR}$ ) for detecting acute kidney injury. AUC, area under the receiver-operating characteristic (ROC) curve; $\mathrm{SE}$, standard error; $\mathrm{Cl}$, confidence interval. tients who underwent major orthopedic surgery in our hospital for over 5 years. The incidence of renal insufficiency after orthopedic surgery has been reported to be up to $9.1 \%[3,4]$. The incidence also differs according to the surgical site. According to a study by the Scottish Arthroplasty Project, the prevalence of AKI after total hip arthroplasty increased from $0.47 \%$ to $0.78 \%$, and the prevalence of AKI after total hip arthroplasty increased from $0.39 \%$ to $0.75 \%$ between 2004 and 2013 [11]. In the present study, the incidence of AKI was 3.7\%, which is low as compared with those recorded in previous studies. The incidence of AKI varies among studies depending on the type of surgery performed [12-14].

In the present study, preoperative hemoglobin levels were found to influence the occurrence of AKI, which is consistent with previous studies [7], thereby suggesting that preoperative general conditions may affect the postoperative clinical course.

In another study, for patients with severe sepsis in the surgical intensive care unit, a lower AKI incidence rate was recorded for a crystalloid group than for a hydroxyethyl starch group [15]. Adequate fluid management with consideration of fluid type is important to maintain hemodynamic stability in patients undergoing surgery. Insufficient fluid administration results in renal hypoperfusion but may cause pulmonary congestion when performed excessively. In the present study, however, we found no statistically significant differences related to the type and amount of fluid administered.

The definition of AKI has been constantly revised and supplemented [16]. We, therefore, sought to identify patients with additional risk of developing AKI according to their $\triangle \mathrm{eGFR}$. A cutoff of 7.54 had a high sensitivity and specificity (100\% and $89.64 \%$, respectively). Therefore, if the $\Delta \mathrm{eGFR}$ is $>7.54$, management is required but still depending on the case. In clinical practice, after checking only creatinine levels, if the change is within the normal range even if the $\triangle \mathrm{eGFR}$ is significant, it is often skipped unremarkably. In our study, $73.3 \%$ of the patients with significant $\Delta \mathrm{eG}$ FR had changes in creatinine level that were within the normal range $(0.7-1.2 \mathrm{mg} / \mathrm{dL})$. Clinicians should check patients' eGFR because if the change is significant, careful monitoring is required particularly for changes in renal function in patients with AKI. These data will help establish the treatment direction and improve prognosis, particularly in older patients, because their treatment can be challenging owing to the difficulty in estimating the accurate renal function using creatinine levels (because of age-related reduction of muscle mass) and other factors [17].

One study showed that blood transfusion, hepaticojejunostomy, and oliguria are the strongest predictors of AKI after liver resection, although their findings could not be applied to orthopedic research because of the specificity of the field [18]. In a cohort study that assessed age, male sex, presence of diabetes, low GFR, use of 
angiotensin-converting enzyme inhibitors or angiotensin receptor blockers, the number of prescriptions, and the American Society of Anesthesiologists grade during surgery for orthopedic patients in Scotland, acute renal injury was identified as a predictor of and confirmed to be associated with increased long-term mortality [19]. In our study, the risk factors considered in previous studies did not show a statistically significant relationship with acute renal injury, and the difference in these results was that the incidence of AKI was lower in our study than in other studies. This could be because our study was conducted in a single race or local area; hence, its main limitation resulted in a selection bias.

This study has some limitations. First, because the study was conducted in a single institution, it was conducted in patients of a single race or from a local area, and a selection bias may be associated with this. Therefore, the results of our study can be applied to domestic patients but will be difficult to apply to various races. Further multicenter studies are needed to augment the information generated from the present study. The second limitation is that it is a retrospective study. Whether the statistically significant variables had a causal relationship with AKI was difficult to confirm, and the association alone could be confirmed. Because the data we used were from patients not recruited for research, new AKI-related risk factors may exist among the variables that we did not consider. Thus, additional prospective studies are needed. The third limitation is a problem because of the small number of patients with AKI as compared with the total number of patients. Owing to the small number of patients, securing statistical significance was difficult. Thus, further studies with larger numbers of patients are needed.

Renal function should be monitored closely after orthopedic surgery if the patient has CKD or low hemoglobin levels, which should be stabilized before the procedure. In addition, clinicians should check the eGFR because a significant change in eGFR requires careful monitoring based on the occurrence of AKI. In elderly patients whose renal functions are difficult to accurately assess on the basis of creatinine levels, more attention should be paid to eGFR changes. Predicting the likelihood of AKI occurrence, early treatment of highrisk patients, and monitoring perioperative laboratory test results, including eGFR, will help improve patient prognosis.

\section{Acknowledgments}

\section{Conflict of interest}

No potential conflict of interest relevant to this article was reported.

\section{Funding}

This study was supported by Inje University Haeundae Paik Hos- pital and a research grant from the Inje University in 2018 (No. 20180167).

\section{Author contributions}

Conceptualization: YWK; Writing-original draft: YJL; Writing-review \& editing: YWK, BSP, SP, JHP, IHK, JK.

\section{ORCID}

Yoo Jin Lee, https://orcid.org/0000-0003-2799-6242

Bong Soo Park, https://orcid.org/0000-0001-8999-386X

Sihyung Park, https://orcid.org/0000-0002-6782-5299

Jin Han Park, https://orcid.org/0000-0002-1138-4957

Il Hwan Kim, https://orcid.org/0000-0003-4166-6303

Junghae Ko, https://orcid.org/0000-0002-0029-6847

Yang Wook Kim, https://orcid.org/0000-0001-9676-5320

\section{References}

1. Chertow GM, Levy EM, Hammermeister KE, Grover F, Daley J. Independent association between acute renal failure and mortality following cardiac surgery. Am J Med 1998;104:343-8.

2. Biteker M, Dayan A, Tekkeşin AI, Can MM, Taycı I, Ilhan E, et al. Incidence, risk factors, and outcomes of perioperative acute kidney injury in noncardiac and nonvascular surgery. Am J Surg 2014;207:53-9.

3. White SM, Rashid N, Chakladar A. An analysis of renal dysfunction in 1511 patients with fractured neck of femur: the implications for peri-operative analgesia. Anaesthesia 2009; 64:1061-5.

4. Paul A, John B, Pawar B, Sadiq S. Renal profile in patients with orthopaedic trauma: a prospective study. Acta Orthop Belg 2009; 75:528-32.

5. Hobson C, Ozrazgat-Baslanti T, Kuxhausen A, Thottakkara P, Efron PA, Moore FA, et al. Cost and mortality associated with postoperative acute kidney injury. Ann Surg 2015;261:120714.

6. Rosner MH, Okusa MD. Acute kidney injury associated with cardiac surgery. Clin J Am Soc Nephrol 2006;1:19-32.

7. Yoshihara H, Yoneoka D. Trends in the incidence and in-hospital outcomes of elective major orthopaedic surgery in patients eighty years of age and older in the United States from 2000 to 2009. J Bone Joint Surg Am 2014;96:1185-91.

8. Statistics Korea. Population projections for Korea: 2017-2067 (based on the 2017 population census) [Internet]. Daejeon: Statistics Korea; 2019 [cited 2020 Jul 1]. http://kosis.kr/publication/publicationThema.do?pubcode $=\mathrm{PJ}$.

9. Kidney Disease: Improving Global Outcomes (KDIGO) Acute 
Kidney Injury Work Group. KDIGO clinical practice guideline for acute kidney injury. Kidney Int Suppl 2012;2:1-138.

10. Kidney Disease: Improving Global Outcomes (KDIGO) CKD Work Group. KDIGO 2012 clinical practice guideline for the evaluation and management of chronic kidney disease. Kidney Int Suppl 2013;3:1-150.

11. Scottish Arthroplasty Project. Biennial report 2014. Edinburgh: NHS National Services Scotland; 2014.

12. Thakar CV. Perioperative acute kidney injury. Adv Chronic Kidney Dis 2013;20:67-75.

13. Sun LY, Wijeysundera DN, Tait GA, Beattie WS. Association of intraoperative hypotension with acute kidney injury after elective noncardiac surgery. Anesthesiology 2015;123:515-23.

14. Lim SY, Lee JY, Yang JH, Na YJ, Kim MG, Jo SK, et al. Predictive factors of acute kidney injury in patients undergoing rectal surgery. Kidney Res Clin Pract 2016;35:160-4.

15. Bayer O, Reinhart K, Sakr Y, Kabisch B, Kohl M, Riedemann NC, et al. Renal effects of synthetic colloids and crystalloids in patients with severe sepsis: a prospective sequential comparison. Crit Care Med 2011;39:1335-42.

16. Levey AS, Eckardt KU, Tsukamoto Y, Levin A, Coresh J, Rossert J, et al. Definition and classification of chronic kidney disease: a position statement from Kidney Disease: Improving Global Outcomes (KDIGO). Kidney Int 2005;67:2089-100.

17. Odden MC, Shlipak MG, Tager IB. Serum creatinine and functional limitation in elderly persons. J Gerontol A Biol Sci Med Sci 2009;64:370-6.

18. Slankamenac K, Beck-Schimmer B, Breitenstein S, Puhan MA, Clavien PA. Novel prediction score including pre- and intraoperative parameters best predicts acute kidney injury after liver surgery. World J Surg 2013;37:2618-28.

19. Bell S, Dekker FW, Vadiveloo T, Marwick C, Deshmukh H, Donnan PT, et al. Risk of postoperative acute kidney injury in patients undergoing orthopaedic surgery: development and validation of a risk score and effect of acute kidney injury on survival: observational cohort study. BMJ 2015;351:h5639. 